\title{
Experimental evidence for a spin-Peierls-transition in $\alpha \prime-\mathrm{NaV}_{2} \mathrm{O}_{5}$
}

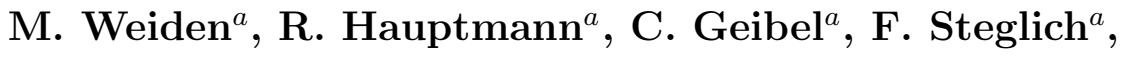 \\ M. Fischer ${ }^{b}$, P. Lemmens ${ }^{b}$, G. Güntherodt ${ }^{b}$ \\ a Technische Physik, TH Darmstadt, Hochschulstr. 8, 64289 Darmstadt, Germany \\ b 2. Physikalisches Institut, RWTH Aachen, Templergraben 55, 52056 Aachen, Germany
}

key-words: spin-Peierls transition, magnetic susceptibility, raman scattering, $\alpha \prime-\mathrm{NaV}_{2} \mathrm{O}_{5}$

\begin{abstract}
We present the first measurements of magnetisation and Raman light scattering on $\alpha \prime-\mathrm{NaV}_{2} \mathrm{O}_{5}$ single crystals. Below $34 \mathrm{~K}$, we observe a pronounced isotropic decrease of the susceptibility indicating the opening of a spin gap. The transition temperature is slightly field dependent. Raman experiments reveal a crystallographic distortion at the transition. Our results clearly establish $\alpha \prime-\mathrm{NaV}_{2} \mathrm{O}_{5}$ to be the second inorganic spin-Peierls system.
\end{abstract}

PACS: 75.30.Kz, 75.50.Ee, 75.40.Cx, 75.90.+w 


\section{INTRODUCTION}

One of the unusual ground states encountered in quasi-one-dimensional spin-systems is the spin-Peierls (SP) groundstate, where a magneto-elastic coupling leads below the transition temperature $T_{S P}$ to a dimerisation of the spin-chain and thus to the formation of a non- magnetic singlet [1]. Up to 1993, this transition has only been observed in a few organic compounds [4]. The discovery of a SP-state in the inorganic compound $\mathrm{CuGeO}_{3}$

[7] has renewed strong interest in this phenomenon: for the first time, detailed studies of a SP-system on large single crystals [8] as well as investigations of the effect of impurities [9] on a SP-groundstate are possible. Yet, despite intensive research for other compounds, $\mathrm{CuGeO}_{3}$ has remained the only known inorganic SP-system. In 1996, Isobe et al. 10 found a pronounced decrease of the susceptibility $\chi(\mathrm{T})$ in $\alpha \prime-\mathrm{NaV}_{2} \mathrm{O}_{5}$ below $\mathrm{T} \approx 34 \mathrm{~K}$ and concluded the opening of a spin-gap. The remaining susceptibility below the transition temperature was attributed to Van-Vleck contributions as observed in $\mathrm{VO}_{2}$, where a singulett is formed already above room temperature [11,12]. The orthorombic crystal structure 13,14 of $\alpha \prime-\mathrm{NaV}_{2} \mathrm{O}_{5}$ contains $V^{4+}(\mathrm{S}=1 / 2)$-chains along the b-axis, in which the spins are antiferromagnetically coupled with an exchange coupling $\mathrm{J}=560 \mathrm{~K} \mathrm{[10]}$. These chains are separated from each other by nonmagnetic $V^{5+}$-chains, which results in a quasi-onedimensional behaviour of the magnetic properties [15,16]. Since Isobe et al. only performed measurements on polycrystalline samples, they were not able to study the orientation dependence of the magnetic transition itself and of the susceptiblity above the transition to proof a negligible influence of spin anisotropy and a primary isotropic Heisenberg-character of the spin-chains. Additionally, they were not able to confirm the existence of any crystallographic distortions at the transition temperature, a feature which is, however, essential for a SP-transition. Also, an analysis of the susceptibility on polycrystalline samples by Mila et al. [16] revealed no transition at all despite a similar exchange coupling of $\mathrm{J}=529 \mathrm{~K}$. 


\section{EXPERIMENTAL DETAILS AND RESULTS}

The single crystals used for the investigations were grown by a self-flux method. Details of the preparation procedure will be published elsewhere [17]. We observe a strong dependence of the transition on the Na-content: both excess and defficiency decrease the transition temperature. Susceptibility measurements were performed with a commercial SQUID- magnetometer (MPMS, Quantum Design). Raman-measurements were performed with an Ar-Laser $(\lambda=514.5 \mathrm{~nm})$ and a Dilor XY-spectrometer including a nitrogen-cooled CCD-detector. The results of the measurements of the susceptibility $\chi(\mathrm{T})$ with magnetic field parallel to the three crystallographic axes are shown in fig. 1. As the crystals are thin platelets, several crystals were oriented parallel and fixed using a small amount of wax. This wax lead to a temperature-independent diamagnetic background, which size could not be determined precisely. Owing to the fact that at low temperatures $\chi(\mathrm{T})$ corresponds to a temperature-independent Van-Vleck-contribution, we shifted the experimental values to fit the value at $\mathrm{T}=2 \mathrm{~K}$ obtained in a measurement without any wax. These shifts correspond to the addition of a temperature-independent value and will therefore neither affect the absolute size of the anomaly in $\chi(\mathrm{T})$ nor the temperature dependence. In contrast to the results of Isobe et al., our measurements show no Curie-contribution at low temperatures, indicating the high quality of the single crystals. The decrease of $\chi(\mathrm{T})$ below the transition temperature presents the same shape and nearly the same magnitude along the three axes. Such an isotropic behaviour is the characteristic sign of the opening of a spin gap and cannot be due to another magnetic instability, e.g. a Né el-transition. The slight differences in the magnitude of $\chi(\mathrm{T})$ above the transition temperature can be accounted for by an anisotropy in the g-factor. Assuming $\mathrm{g}=2$ for $\mathrm{B} \| \mathrm{c}$, this would lead to $\mathrm{g}=2.06$

for $\mathrm{B} \| \mathrm{b}$ and $\mathrm{g}=2.14$ for $\mathrm{B} \|$ a. Apart for this difference, the temperature dependence of $\chi(\mathrm{T})$ is the same along the three axes indicating the isotropic Heisenberg-character of the spin-chains. Below the transition temperature, the temperature dependence of the susceptibility was fitted using the theory of Bulaevskii [18], where $\chi(\mathrm{T})$ of a dimerized chain is 
calculated using two parameters $\mathrm{J}$ and $\gamma$, J being the mean coupling constant, $\gamma$ describing the dimerization. The gap energy $\Delta$ can be deduced from $\mathrm{J}$ and $\gamma$ using the theories of Pytte [19] and Bray et al. [4]. The result of the fit is shown in the inset of fig. 1. We obtain $\Delta \approx 85115 \mathrm{~K}, \mathrm{~J}=441 \mathrm{~K}$; the values differ only slightly between the different directions and for similar analyses on polycrystals. $\mathrm{J}$ is in good agreement with $\mathrm{J}=560$ $\mathrm{K}[10]$ or $\mathrm{J}=529 \mathrm{~K}[16]$ obtained from the high temperature susceptibility. Investigations of the field dependence of the transition temperature of a polycrystalline sample revealed a small shift to lower temperatures $\Delta T \approx 0.15 \mathrm{~K}$ at $\mathrm{B}=5.5 \mathrm{~T}$. The observed field dependence can be well described using the theory of Bulaevskii [18] and Cross [2] for the field dependence of a SP-system: $T_{S P}(0) / T_{S P}(B)-1=\alpha\left[\left(g \mu_{B} B\right) /\left(k_{B} T_{S P}(0)\right)\right]^{2}$. We found $\alpha \approx$ 0.118, in good agreement with theoretical predictions of $\alpha=0.091-0.11$ for a SP-system [1.2]. This field dependence clearly indicates that the transition is not a simple structural one. Raman scattering experiments were performed in quasi-backscattering geometry. The polarization was parallel to the $V^{4+}$-chains (bb), perpendicular (aa) or crossed polarized (ab). Several phonons could be detected for temperatures above the transition temperature. Six modes were observed both in (aa) as well as in (bb) geometry: at 88, 178, 304, 420, 530 and $971 \mathrm{~cm}^{-1}$. Additionaly there is one mode at $230 \mathrm{~cm}^{-1}$ only observable in (aa) and one mode at $449 \mathrm{~cm}^{-1}$ only observable in (bb)-polarization. In (ab) geometry, four modes were identified at 173, 262, 292 and $685 \mathrm{~cm}^{-1}$. Besides, small residual contributions could be observed at 449 and $530 \mathrm{~cm}^{-1}$. Along the chains (b-axis), the modes at 449 and 530 $\mathrm{cm}^{-1}$ are strongly asymmetric and show a tail towards the high energy side. Taking into account that the energy of these modes are very close to the energy scale of spin excitations along the Heisenberg chains, we would suggest that these tails form due to magnetic Raman scattering. Further experiments are required to clarify the nature of this effect. Below the transition temperature several new modes could be detected. In Fig. 2, spectra at $\mathrm{T}=100$ $\mathrm{K}$ and at $\mathrm{T}=5 \mathrm{~K}$ in (bb) geometry are compared. Strong additional peaks are seen at $65,104,130,650$ and $945 \mathrm{~cm}^{-1}$. In addition, smaller contributions appear at 230, 246 and $296 \mathrm{~cm}^{-1}$. Also, a formerly symmetry forbidden mode from (ab)-polarization at $685 \mathrm{~cm}^{-1}$ 
is now observable. As e.g. the mode at $945 \mathrm{~cm}^{-1}$ can be asssigned to a phonon mode due to its lineshape, clear evidence is given that the transition leads to a symmetry breaking of the lattice.

\section{CONCLUSION AND SUMMARY}

We have grown for the first time single crystals of $\alpha \prime-\mathrm{NaV}_{2} \mathrm{O}_{5}$ and present the first investigations of the orientation-dependence of the susceptibility and the first Raman-results. We found at the transition temperature an isotropic decrease of the susceptibility pointing to the opening of a spin-gap and the formation of a spin-singlet. Also, the isotropy of the susceptibility proves the chains to be of isotropic Heisenberg-type. The Raman experiments indicate that this magnetic transition is accompanied by a crystallographic distortion. The field dependence of the transition temperature rules out a simple structural transition. The isotropic exponential disappearance of the spin-susceptibility, a simultaneous crystallographic distortion and the field dependence of the transition temperature are the hallmarks

of a spin-Peierls transition. Therefore, our results clearly prove $\alpha \prime-\mathrm{NaV}_{2} \mathrm{O}_{5}$ to be the second inorganic SP-system, having the highest transition temperature $\mathrm{T}_{S P}=34 \mathrm{~K}$ of all known SP-systems.

Acknowledgment: This work was supported by SFB 252, SFB 341 and BMBF FKZ.13N6586 We acknowledge the help of P. Haberl in using the Ljour2-stylefile. 


\section{REFERENCES}

[1] Bulaevskii, L.N.: Soviet Phys. - Solid State 11, 921 (1969)

[2] Cross, M.C.: Phys. Rev. B 20, 4606 (1979)

[3] Khomskii, D., Geertsma, W., Mostovoy, M.: Czech. J. Phys. S5, (1996)

[4] Bray, J.W., Hart, H.R., Interrante, L.V.Jr., Jacobs, I.S., Kasper, J.S., Watkins, G.D., Wee, S.H., Bonner, J.C.: Phys. Rev. Lett. 35, 744 (1975)

[5] Huizinga, S., Kommandeur, J., Sawatzky, G.A., Thole, B.T., Kopinga, K., deJonge, W.J.M., Roos, J.: Phys. Rev. B 19, 4723 (1979)

[6] Jacobs, I.S., Bray, J.W., Hart, H.R., Interrante, L.V.Jr., Kasper, J.S., Watkins, G.D., Prober, D.E., Bonner, J.C.: Phys. Rev. B 14, 3036 (1976)

[7] M. Hase, I. Terasaki, K. Uchinokura, Phys. Rev. Lett. 70, 3651 (1993)

[8] Boucher, J.P., Regnault, L.P.: submitted to J. de Phys.

[9] M. Weiden, W. Richter, R. Hautmann, C. Geibel, F. Steglich, accepted by Physica B, and references therein

[10] M. Isobe, Y. Ueda, J. Phys. Soc. Jap. 65, 1178 (1996)

[11] J.B. Goodenough, Progress in Solid State Chemistry 5, 145, Pergamon Press New York (1971)

[12] T. Kawakubo, T. Nakagawa, J. Phys. Soc. Jap. 19, 517 (1964)

[13] M. Pouchard, A. Casalot, J. Galy, P. Hagenmuller, Bull. Soc. Chim. France 11, 4343 $(1967)$

[14] A. Carpy, J. Galy, Acta Cryst. B 31, 1481 (1975)

[15] A. Carpy, A. Casalot, M. Pouchard, J. Galy, P. Hagenmuller, J. Sol.State Chem. 5, 229 $(1972)$ 
[16] Mila, F., Millet, P., Bonvoisin, J.: preprint cond-mat/9605024 v2

[17] M. Fischer, P. Lemmens, G. Güntherodt, M. Weiden, R. Hauptmann, C. Geibel, F. Steglich, to be published

[18] Bulaevskii, L.N., Buzdin, A.I., Khomskii, D.I.: Sol. State Comm. 27, 5 (1978)

[19] Pytte, E.: Phys. Rev. B 10, 4637 (1974) 


\section{FIGURES}

FIG. 1. Susceptibility of $\alpha \prime-\mathrm{NaV}_{2} \mathrm{O}_{5}$ along the three major crystallographic axes. The values for the magnetic field parallel to the three axes are shifted to reach the same value at $\mathrm{T}=2 \mathrm{~K}$ (see text). At the transition temperature, an isotropic decrease is observed, which proves the opening of a spin gap. Inset: Analysis of the susceptibility of the single crystals for B $\|$ b using the theory of Bulaevskii. The solid line represents the fit with $\Delta \approx 85 \mathrm{~K}, \mathrm{~J}=441 \mathrm{~K}$.

FIG. 2 Raman measurements above and below the transition temperature in (b,b)-polarisation. Below the transition, several additional modes are observed. 


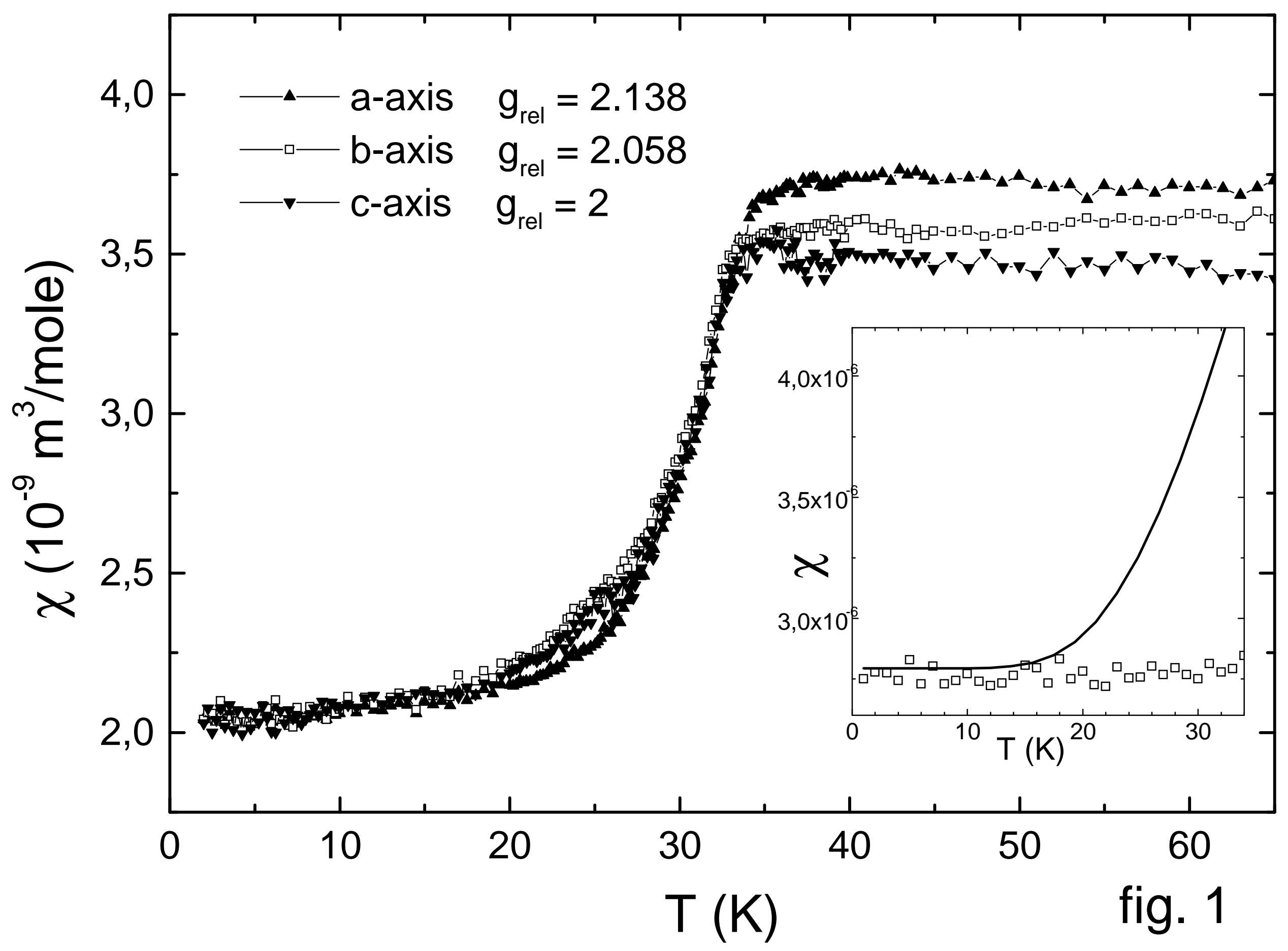




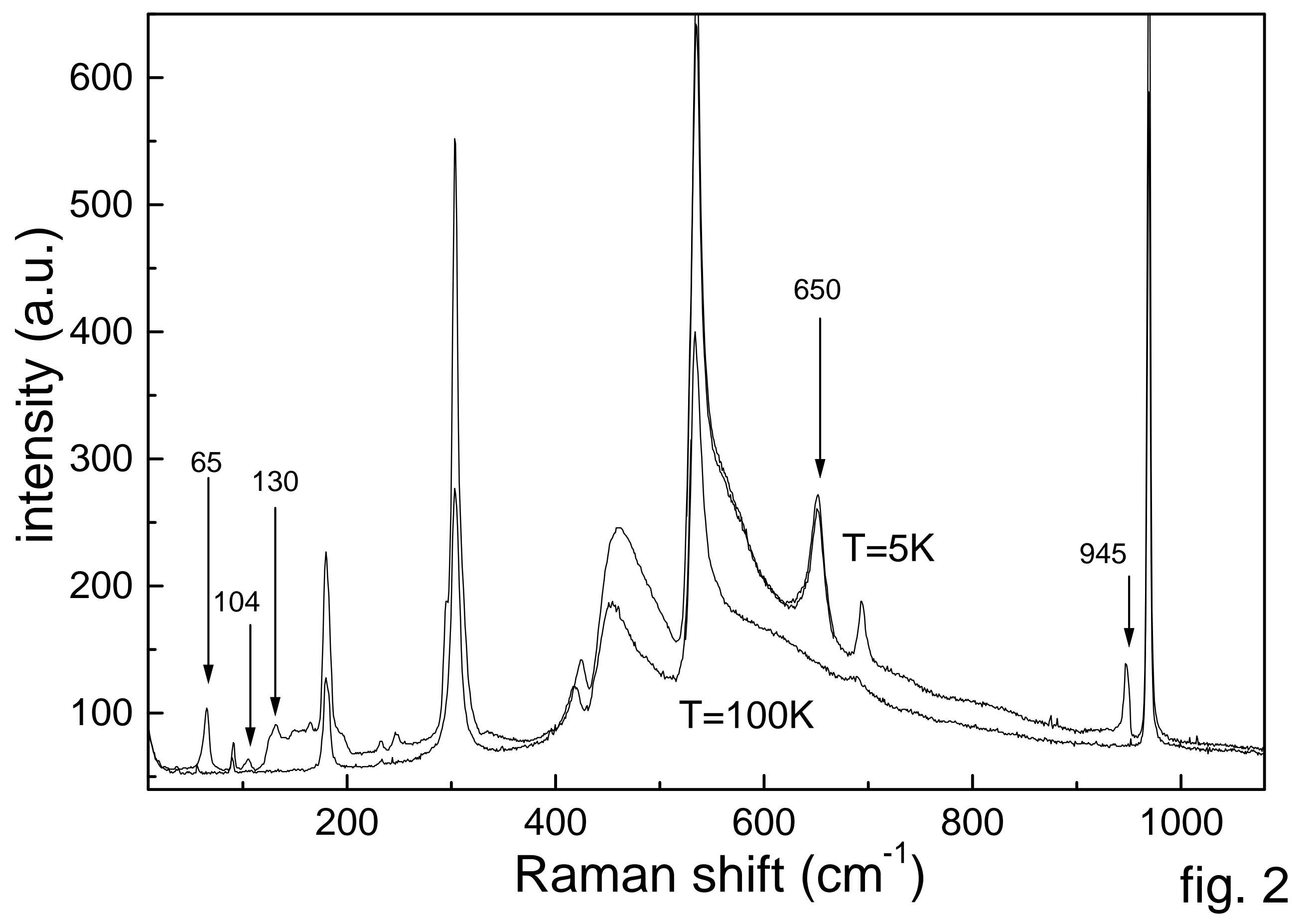

